\title{
03 THIRTY DAY OUTCOMES OF SEVERE INJURIES IN PATIENTS PRESENTING TO THE EMERGENCY DEPARTMENTS OF TWO PUBLIC TERTIARY CARE HOSPITALS IN SINDH PAKISTAN
}

doi:10.1136/injuryprev-2012-040590b.3

${ }^{1}$ J. Razzak, ${ }^{2}$ S. Kalhoro. ${ }^{1}$ Aga Khan University, Pakistan; ${ }^{2}$ Save the Children, Pakistan

Background Post traumatic mortality and morbidity (PTM\&M) is different between different designated hospitals so studies should be done to assess the difference in PTM\&M between similar designated hospitals

Objectives To assess the difference in the rate of death and disability at 30 -days among patients aged $\geq 14-80$ years with history of severe injuries presenting at ER of two public tertiary care hospitals in Sindh Pakistan. To identify risk factors for death and disability due to severe injuries among patients aged $\geq 14-80$ year presenting to the ER of two public tertiary care hospitals in Sindh Pakistan.

\section{Methodology}

Study design: longitudinal cohort

Sample size: 390 eligible participants

Study follow-up: 30 days

Study site: civil hospital Karachi and Hyderabad

Data collection tools: Baseline Questionnaire and SF 36 for disability

Statistical analysis: Rate ratio, rate difference and logistic regression

Results By taking Hyderabad as reference, there was no significant difference in incidence rate of mortality (rate ratio 1.12 (95\% CI 0.77 to 1.63$)$ ) (rate difference $14(-33,61)$ ), incidence rate of physical disability (rate ratio 1.01 (95\% CI 0.67 to 1.51$)$ ) (rate difference $0.88(-44,46))$ and incidence rate of mental disability (rate ratio 0.69 (95\% CI 0.40 to 1.21$)$ ) (rate difference -220 (95\% CI -556 to 115)) injury severity, patients attended by paramedics as compared to doctors in the ER and delay to receive general management were associated with mortality. Lower extremity injury and higher hospital stay were associated with worse disability.

Conclusion Incidence rates of mortality and disability were not different between two tertiary care settings but overall incidence rate of death and disability was higher in severely injured 